\title{
Microcavity two-unit tandem organic light-emitting devices having a high efficiency
}

\author{
Ting-Yi Cho, Chun-Liang Lin, and Chung-Chih $\mathrm{Wu}^{\mathrm{a})}$ \\ Department of Electrical Engineering, Graduate Institute of Electro-optical Engineering, \\ and Graduate Institute of Electronics Engineering, National Taiwan University, Taipei, \\ Taiwan 10617, Republic of China
}

(Received 16 November 2005; accepted 24 January 2006; published online 14 March 2006)

\begin{abstract}
In pursuit of further enhancement in luminance and efficiency of organic light-emitting devices (OLEDs), it is worth exploring what benefits could be obtained by combining two luminance-enhancement techniques, i.e., microcavity and tandem OLEDs. In this letter, we have investigated theoretically and experimentally the characteristics of noncavity and microcavity tandem OLEDs. Results show that with well designed microcavity and device structures (i.e., consistent with resonant and antinode conditions), a fivefold enhancement in luminance can be achieved with cavity tandem devices having only two emitting units. A very high efficiency of $200 \mathrm{~cd} / \mathrm{A}$ has been demonstrated with a phosphorescent cavity two-unit device. (C) 2006 American Institute of Physics. [DOI: 10.1063/1.2185077]
\end{abstract}

Rapid advances of organic light-emitting devices (OLEDs) and their applications in displays and lighting impose substantial demands in OLED structures having enhanced brightness yet without increasing driving currents. ${ }^{1,2}$ Two techniques associated with such demands are thus developed in parallel in recent years. Tandem OLEDs provide enhanced luminance and current (cd/A) efficiency through stacking multiple emitting units vertically in series. ${ }^{3,4}$ On the other hand, incorporating well designed microcavity structures in OLEDs can provide up to twofold enhancement in luminance and cd/A efficiency through redistributing radiation generated in devices. ${ }^{5-7}$ In pursuit of further enhancement in luminance and efficiency of OLEDs, it is worth further exploring what benefits could be obtained by combining both techniques, i.e., by forming microcavity tandem OLEDs. In this letter, we investigate theoretically and experimentally the characteristics of microcavity tandem OLEDs. Results show that by incorporating the microcavity structure, very high efficiency $(200 \mathrm{~cd} / \mathrm{A})$ can be achieved with a tandem OLED having only two emitting units.

Noncavity and cavity tandem OLEDs for analyses and experiments are green phosphorescent OLEDs based on the emitter tris(phenylpyridine)iridium $\left(\operatorname{Ir}(\mathrm{ppy})_{3}\right) .^{8}$ Noncavity tandem OLEDs have the typical bottom-emitting structure with a transparent indium-tin-oxide (ITO) anode on glass substrates and a high-reflectivity Ag cathode. The cavity tandem OLEDs adopt the top-emitting configuration with the high-reflectivity $\mathrm{Ag}$ as the bottom anode and the semitransparent $\mathrm{Ag}(20 \mathrm{~nm})$ as the top cathode. According to previous studies of microcavity OLEDs, ${ }^{5,7}$ for maximizing output luminance of cavity OLEDs, the thin $\mathrm{Ag}$ is further capped with a $60 \mathrm{~nm}$ high-index organic layer $\left[4,4^{\prime}, 4^{\prime \prime}\right.$-tris(N-carbazolyl)-triphenylamine, TCTA $]$ to achieve both high reflection (seen from the inside of the device) and low absorption for major emission wavelengths of $\operatorname{Ir}(\mathrm{ppy})_{3}$, and the total thickness of organic layers between electrodes is adjusted to achieve the lowest possible resonance mode (for $530 \mathrm{~nm}$ ) for each tandem device. To sim-

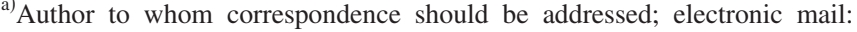
chungwu@cc.ee.ntu.edu.tw plify calculation, an average refractive index of 1.77 over wavelengths of interest is assumed for all organic layers in the devices. The optical model used for performing the analysis adopts a classical approach based on the equivalence between the emission of a photon due to an electrical dipole transition and the radiation from a classical electrical dipole antenna, ${ }^{9-11}$ which can take into account loss due to electrodes. With plane-wave expansion of the dipole field, the full-vectorial electromagnetic fields generated by a radiation dipole embedded in a layered structure is calculated, from which the distribution of the radiation power into different plane-wave modes and the far-field radiation related to emission characteristics of an OLED are obtained. To model emission characteristics of an OLED, it is assumed that the emitting layer contains an ensemble of mutually incoherent dipole radiators with distributions in dipole orientations (a random isotropic distribution), locations (throughout the $15 \mathrm{~nm}$ emitting layer), and frequencies [using the photoluminescence (PL) spectrum of $\operatorname{Ir}(\mathrm{ppy})_{3}$ as the intrinsic spectral distribution of the dipole radiators]. Radiation characteristics of OLEDs are then obtained by averaging contributions over these distributions.

Figures 1(a)-1(c) show the calculated forward luminance resulting from a single emitting layer located at different distances to the back mirror electrode in one-, two-, and three-unit noncavity tandem devices, respectively, along with the fraction of radiation coupled into plasmon modes and waveguided modes (including both substrate and ITO/ organic modes). Similarly, Figs. 1(d)-1(f) show those for cavity tandem devices. In all cases, the forward luminance is normalized to that of the optimized one-unit bottom-emitting OLED (i.e., the reference device). In these results, the maxima occurring in forward luminance roughly correspond to antinodes of the metal electrodes (i.e., the emitter-to-metal round-trip phase change equals to integral multiples of $2 \pi$ ), while minima correspond to nodes. Interestingly, emitters located beyond the first antinode of noncavity tandem OLEDs or at antinodes of cavity devices in general contribute larger forward luminance (up to 1.5 times in the noncavity device and 2.6 times in the cavity device) than the reference device. Such enhancement in some cases is partly associated with 

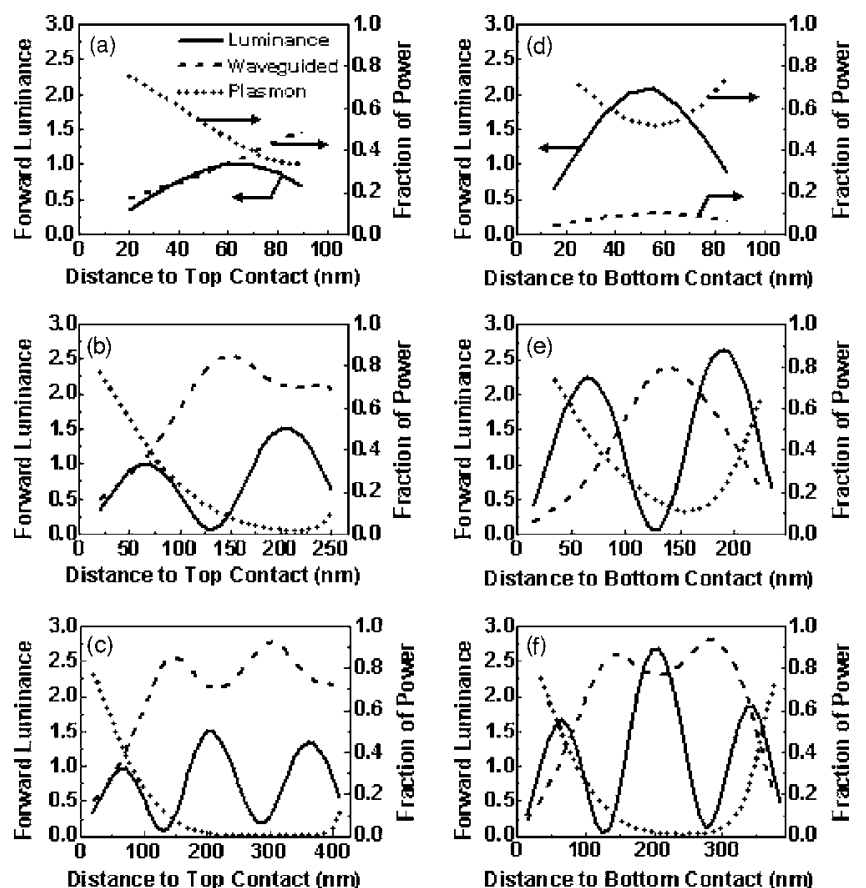

FIG. 1. Calculated forward luminance and fractions of radiation coupled into plasmon modes and waveguided modes (including both substrate and ITO/organic modes for the case of noncavity devices) resulting from an emitting layer located at different distances to the back mirror electrode in noncavity one-unit (a), two-unit (b), and three-unit (c) tandem devices ( $\sim 2 \%-3 \%$ absorption loss in ITO not shown), and in microcavity one-unit (d), two-unit (e), and three-unit (f) tandem devices ( $\sim 10 \%$ absorption loss in the top electrode not shown). In all figures, the forward luminance is normalized to that of the optimized one-unit noncavity device (i.e., the reference device), and the left and right ends of the horizontal axes represent interfaces to electrodes, respectively.

enhanced outcoupling (considering ratios of plasmon modes and waveguided modes etc.), such as the cases of farther antinodes in noncavity tandem devices (negligible plasmon modes) and the case of the one-unit cavity device (low coupling into waveguided modes). In most cases, however, such enhanced forward luminance is more associated with stronger coupling of internal radiation into the forward direction (i.e., more directed emission) according to detailed analyses, paticularly for cavity devices where strongly directed emission leads to higher forward luminance than in noncavity devices. In a same tandem device, emitters located around the antinode closest to a metal electrode suffer strong coupling into plasmon modes and thus lower outcoupling and forward luminance. In contrast, locating emitters at farther antinodes reduces plasmon modes to nearly negligible levels; although coupling into waveguided modes also increases, yet in general stronger outcoupling and forward luminance are obtained. Such characteristics may explain the observations in few reported tandem devices that the enhancement in $\mathrm{cd} / \mathrm{A}$ efficiency could indeed significantly exceed proportional increase with the number of emitting units. ${ }^{3,4}$ For both tandem devices, with increasing the number of emitting units (and thus the total thickness of devices), overall coupling into wavegudied modes rises and eventually will diminish advantages of tandem devices.

Based on results of Fig. 1, a clear design rule for tandem OLEDs (either noncavity or cavity) is to locate emitters of each emitting unit at corresponding antinodes. Summing the peak values of forward luminance for each device in Fig. 1 gives the optimal overall luminance enhancement (relative to Downloaded 17 Feb 2009 to 140.112 .113 .225 . Redistribution subje
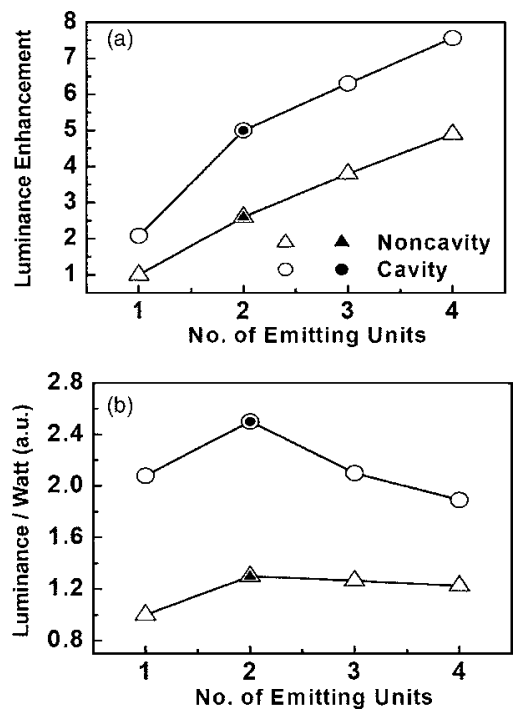

FIG. 2. (a) Calculated luminance enhancement (open symbols) vs the number of emitting units, and (b) calculated luminance/watt (open symbols, normalized to that of the reference device) for both noncavity and cavity tandem devices. Experiment results (solid symbols) are also shown in (a) and (b) for comparison.

the reference one-unit device) for each tandem device. Figure 2(a) shows the luminance enhancement versus the number of emitting units for both types of tandem devices. In general, luminance increases with the number of emitting units, but not linearly due to larger loss into waveguided modes for thicker devices. The deviation is even larger for cavity devices for mode widths narrow with cavity lengths. ${ }^{5,6}$ In tandem devices, not only brightness but also the operation voltage increases with the number of units. Thus a fair way to characterize performances of tandem devices is to evaluate their luminance per electrical wattage. Assuming linear increase of operation voltage, Fig. 2(b) shows such characteristics of tandem devices (normalized to that of the reference device). Noncavity tandem devices exhibit rather constant enhancement of $\sim 1.2-1.3$, while an enhancement of $\sim 2.1$ and an optimal enhancement of $\sim 2.5$ are obtained with oneand two-unit cavity devices, respectively.

Experiments were conducted on the reference device (A), noncavity (B), and cavity (C) two-unit devices (Fig. 3) for comparison with analyses. Device A had the structure of: glass/ITO $(120 \mathrm{~nm}) / m$-MTDATA: $\mathrm{F}_{4}-\mathrm{TCNQ} \quad(2 \mathrm{wt} \%, 20 \mathrm{~nm}) /$ $\alpha$-NPD: $\mathrm{F}_{4}-\mathrm{TCNQ}(2$ wt $\%, 8 \mathrm{~nm}) / \alpha$-NPD $(10 \mathrm{~nm}) /$ TCTA

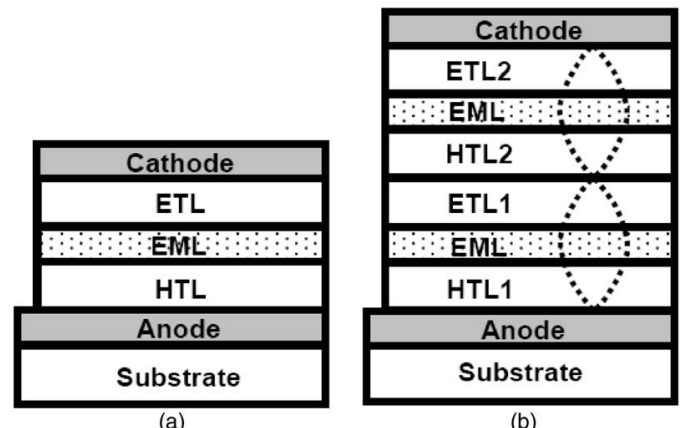

FIG. 3. Schematic device structures of (a) the reference noncavity one-unit device (device $\mathbf{A}$ ) and (b) the two-unit tandem devices (noncavity device B and cavity device $\mathbf{C}$ ). Dotted lines in (b) schematically represent the distribution of the electric-field intensity in the device.

to AIP license or copyright; see http://apl.aip.org/apl/copyright.jsp 

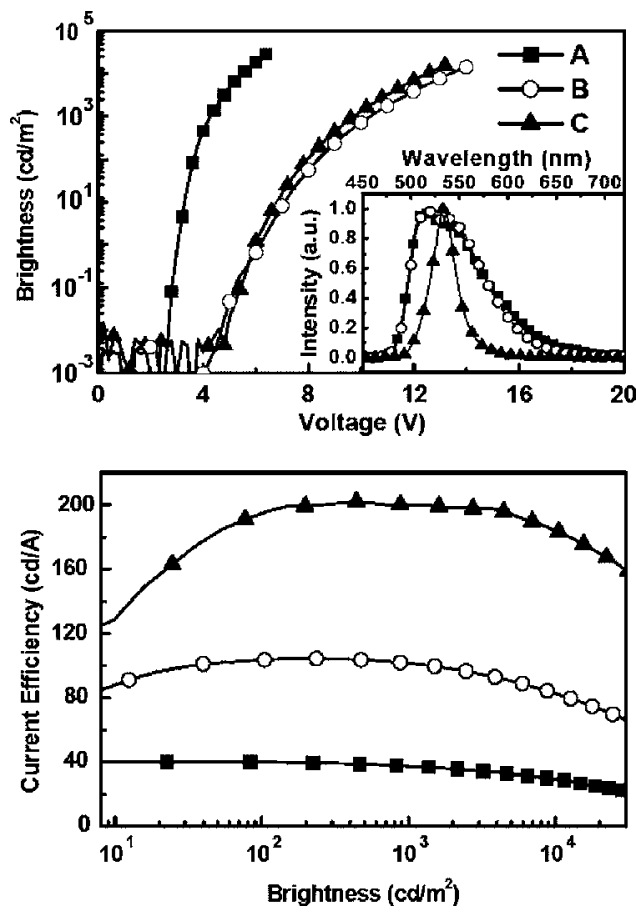

FIG. 4. (a) Brightness-voltage $(B-V)$ characteristics, and (b) the cd/A efficiency vs brightness of devices $\mathbf{A}, \mathbf{B}$, and $\mathbf{C}$. Inset of (a) shows the EL spectra of the three devices.

$\left(\begin{array}{ll}10 & \mathrm{~nm}\end{array}\right) / \mathrm{TCTA}: \operatorname{Ir}(\mathrm{ppy})_{3} \quad(8 \mathrm{wt} \%, 5 \mathrm{~nm}) / \mathrm{TAZ}: \operatorname{Ir}(\mathrm{ppy})_{3}$ $(8$ wt $\%, 10 \mathrm{~nm}) / \mathrm{TAZ}(10 \mathrm{~nm}) / \mathrm{BPhen}(10 \mathrm{~nm}) /$ BPhen:Cs $(20 \mathrm{~mol} \%, 27 \mathrm{~nm}) / \mathrm{Al} \quad(1 \quad \mathrm{~nm}) / \mathrm{Ag} \quad(150 \quad \mathrm{~nm})$, which uses conductive doping in carrier-transport layers for current conduction and injection. Organic layers in sequence consist of hole-transport layers (HTL) 4, 4', 4"-tris(N-3-methylphenyl-N-phenyl-amino) - triphenylamine (m-MTDATA) and N,N'-Di(naphthalen-1-yl)-N, $\mathrm{N}^{\prime}$-diphenyl-benzidine ( $\alpha$-NPD) with 2,3,5,6-tetrafluoro7,7,8,8-tetracyanoquinodimethane $\left(\mathrm{F}_{4}-\mathrm{TCNQ}\right)$ as $p$-type conductive doping, ${ }^{4,12}$ intrinsic hole-transport layers $\alpha$-NPD and TCTA, double emissive layers (EML) TCTA, and 3-(4Biphenylyl)-4-phenyl-5-tert-butylphenyl-1,2,4-triazole (TAZ) doped with $\operatorname{Ir}(\text { ppy })_{3},{ }^{13}$ intrinsic electron-transport layers (ETL) TAZ and 4,7-diphenyl-1,10-phenanthroline (BPhen), and BPhen with Cs as $n$-type conductive doping. ${ }^{13}$ Intrinsic organic layers are inserted between emission and conductive transport layers to reduce quenching associated with conductive doping., ${ }^{3,4,12,13}$ Based on device $\mathbf{A}$, doubleunit tandem devices $\mathbf{B}$ and $\mathbf{C}$ (Fig. 3) were fabricated with the structures of: anode $/ m$-MTDATA: $\mathrm{F}_{4}-\mathrm{TCNQ}(20 \mathrm{~nm})$ $/ \alpha$-NPD: $\mathrm{F}_{4}-\mathrm{TCNQ}(10 \mathrm{~nm}) / \alpha$-NPD $(10 \mathrm{~nm}) / \mathrm{TCTA}(10$ $\mathrm{nm}) / \mathrm{TCTA}: \operatorname{Ir}(\mathrm{ppy})_{3}(5 \mathrm{~nm}) / \mathrm{TAZ}: \operatorname{Ir}(\mathrm{ppy})_{3}(10 \mathrm{~nm}) / \mathrm{TAZ}(10$ $\mathrm{nm}) / \mathrm{BPhen}(10 \mathrm{~nm}) / \mathrm{BPhen}: \mathrm{Cs}(40 \mathrm{~nm}) / \alpha$-NPD: $\mathrm{F}_{4}$-TCNQ $(40 \mathrm{~nm}) / \alpha$-NPD $(10 \mathrm{~nm}) /$ TCTA $(10 \mathrm{~nm}) / \mathrm{TCTA}: \operatorname{Ir}(\text { ppy })_{3}(5$ $\mathrm{nm}) / \mathrm{TAZ}: \operatorname{Ir}(\mathrm{ppy})_{3}(10 \mathrm{~nm}) / \mathrm{TAZ}(10 \mathrm{~nm}) / \mathrm{BPhen}(10 \mathrm{~nm})$ /BPhen:Cs $(40 \mathrm{~nm}) /$ cathode. The noncavity device $\mathbf{B}$ used ITO and $\mathrm{Al}(1 \mathrm{~nm}) / \mathrm{Ag}(150 \mathrm{~nm})$ as the anode and the cathode, respectively, while the cavity device $\mathbf{C}$ used $\mathrm{Ag}$ $(100 \mathrm{~nm})$ and $\mathrm{Al}(1 \mathrm{~nm}) / \mathrm{Ag}(20 \mathrm{~nm}) /$ TCTA $(60 \mathrm{~nm})$ as the bottom reflective anode and the semitransparent top cathode. Both tandem devices employed the doped $p$ - $n$ junction to effectively connect two units. ${ }^{4}$ Thicknesses of conductive organic layers were adjusted to match the antinode and resonance conditions.

Figure 4 shows the brightness-voltage $(B-V)$ and the ef- ficiency characteristics of these devices. Reference device A shows steep $B-V$ characteristics and low operation voltages typical for OLEDs with conductive doping. ${ }^{3-5,12,13}$ Noncavity and cavity tandem devices $\mathbf{B}$ and $\mathbf{C}$ show operation voltages roughly doubling that of device $\mathbf{A}$, indicating effectiveness of the conductive $p-n$ junction (i.e., BPhen:Cs/ $\alpha$-NPD: $\left.\mathrm{F}_{4}-\mathrm{TCNQ}\right)$ in electrically connecting two emitting units. With stacking two emitting units and locating emitters around the antinodes, noncavity tandem device $\mathbf{B}$ achieves maximal efficiencies of $105 \mathrm{~cd} / \mathrm{A}$ and $29 \%$ photon/electron (roughly 2.4-2.6× device A efficiencies (40 cd/A, 12\%)). By incorporating the tandem structure into the cavity, device C shows a maximal efficiency of $200 \mathrm{~cd} / \mathrm{A}$, roughly $2 \times$ device $\mathbf{B}$ efficiency and $5 \times$ device $\mathbf{A}$ efficiency. The quantum efficiency of device $\mathbf{C}(28 \%)$ is similar to that of device B. In Figs. 2(a) and 2(b), measured efficiency enhancement of devices $\mathbf{B}$ and $\mathbf{C}$ (solid symbols) are compared with calculated ones (open symbols). Fairly good agreement indicates consistency with analyses. The inset of Fig. 4(a) shows electroluminescence (EL) spectra of the three devices, in which EL of device $\mathbf{C}$ is narrowed due to strong microcavity effects. Since the forward resonant wavelength of the microcavity device is set near the peak wavelength of the PL spectrum, the blueshift of EL spectra with viewing angles is bounded by the shorter-wavelength falloff of PL and thus is slight. ${ }^{11,14}$

In summary, we have investigated theoretically and experimentally the emission characteristics of noncavity and microcavity tandem OLEDs. Results show that with well designed microcavity and device structures (i.e. consistent with resonant and antinode conditions), a fivefold enhancement in luminance can be achieved with cavity tandem devices having only two emitting units, and a very high efficiency of $200 \mathrm{~cd} / \mathrm{A}$ is demonstrated with a phosphorescent microcavity two-unit device.

The authors would like to acknowledge financial support from the National Science Council of Republic of China. C.-L.L. is also grateful for financial support from a MediaTek Fellowship.

${ }^{1}$ C. W. Tang and S. A. VanSlyke, Appl. Phys. Lett. 51, 913 (1987).

${ }^{2}$ C. W. Tang, S. A. VanSlyke, and C. H. Chen, Appl. Phys. Lett. 65, 3610 (1989).

${ }^{3}$ T. Matsumoto, T. Nakada, J. Endo, K. Mori, N. Kavamura, A. Yokoi, and J. Kido, Proceedings 2003 Society for Information Display (SID) International Symposium, Baltimore, MD, p. 9792003.

${ }^{4}$ L. S. Liao, K. P. Klubek, and C. W. Tang, Appl. Phys. Lett. 84, 167 (2004).

${ }^{5}$ C.-L. Lin, H.-W. Lin, and C.-C. Wu, Appl. Phys. Lett. 87, 021101 (2005).

${ }^{6}$ R. H. Jordan, L. J. Rothberg, A. Dodabalapur, and R. E. Slusher, Appl. Phys. Lett. 69, 1997 (1996).

${ }^{7}$ M. H. Lu, M. S. Weaver, T. X. Zhou, M. Rothman, R. C. Kwong, M. Hack, and J. J. Brown, Appl. Phys. Lett. 81, 3921 (2002).

${ }^{8}$ M. A. Baldo, S. Lamansky, P. E. Burrows, M. E. Thompson, and S. R. Forrest, Appl. Phys. Lett. 75, 4 (1999).

${ }^{9}$ K. Neyts, J. Opt. Soc. Am. A 15, 962 (1998).

${ }^{10}$ W. Lukosz, J. Opt. Soc. Am. 67, 1607 (1977).

${ }^{11}$ C.-C. Wu, C.-L. Lin, P.-Y. Hsieh, and H.-H. Chiang, Appl. Phys. Lett. 84, 3966 (2004).

${ }^{12}$ X. Zhou, M. Pfeiffer, J. Blochwitz, A. Werner, A. Nollau, T. Fritz, and K. Leo, Appl. Phys. Lett. 78, 410 (2001).

${ }^{13}$ G. He, M. Pfeiffer, K. Leo, M. Hofmann, J. Birnstock, R. Pudzich, and J. Salbeck, Appl. Phys. Lett. 85, 3911 (2004).

${ }^{14}$ C.-C. Wu, C.-W. Chen, C.-L. Lin, and C.-J. Yang, J. Display Tech. 1, 248 (2005).

Downloaded 17 Feb 2009 to 140.112.113.225. Redistribution subject to AIP license or copyright; see http://apl.aip.org/apl/copyright.jsp 